\title{
Primary Care, Depression, and Anxiety: Exploring Somatic and Emotional Predictors of Mental Health Status in Adolescents
}

\author{
Ian P. Dumont, BA, and Ardis L. Olson, MD
}

Introduction: A growing body of research points to regular, comprehensive mental health screening in primary care practices as an effective tool, but a thorough and efficient approach is not yet widely used. The purpose of this report is to describe the pattern of mental health-related concerns, protective and social risk factors reported by adolescents during routine well-child visits in primary care settings, and their occurrence among teens that screen positive for either depression or anxiety with brief validated measures.

Methods: A personal digital assistant-based questionnaire was administered as part of clinical care to adolescents 11 to 18 years old $(N=2184)$ attending preventive well-child visits in 13 pediatric and family medicine primary care practices in a northern New England practice-based research network over 18 months (2008 to 2009). Depressive and anxiety-related symptoms were assessed using the 2-question versions of the Patient Health Questionnaire and Generalized Anxiety Disorder scale, respectively. Analyses determined the role that the protective and social risk factors played in determining who screens positive for depression and anxiety.

Results: In the fully adjusted model, risk factors that were significant $(P<.05)$ predictors for a positive screen of depression included substance use (adjusted odds ratio [AOR], 2.05); stress (AOR, 3.59); anger (AOR, 1.94); and worries about family alcohol and drug use (AOR, 2.69). Among protective factors, that is, those that reduce the risk of depression, age (AOR, 0.87 for younger patients); having parents who listen (AOR, 0.34); and having more assets (AOR, 0.65) were significant. Significant predictors of screening positive for anxiety included substance use (AOR, 1.97); stress (AOR, 6.10); anger (AOR, 2.31); trouble sleeping (AOR, 1.75), and the sex of the adolescent (A0R, 1.87 for girls). Although having parents who listen was still a significant protective factor for anxiety (AOR, 2.26), other assets were not significant.

Conclusions: Comprehensive primary care mental health screening that considers both anxiety and depression while including strength-based and psychosocial support questions is a helpful adjunct to clinical practices and has been done routinely by using an electronic tool at the point of care. Because certain common somatic and emotional concerns can precede depression and anxiety, routine screening for these issues along with depression and anxiety screening is suggested. (J Am Board Fam Med 2012; 25:291-299.)

Keywords: Adolescent, Anxiety, Depression, Mental Health, Pediatrics, Screening

Adolescence is a time of emerging risky health behaviors, and many adult mental health disorders have their onset during this time period. The median age of onset of adult depression occurs at age

This article was externally peer reviewed.

Submitted 15 February 2011; revised 24 October 2011; accepted 31 October 2011.

From Children's Hospital, Boston, MA (IPD); and the Departments of Pediatrics and Community and Family Medicine, Dartmouth Hitchcock Medical Center, Lebanon, NH (ALO).
14 and anxiety disorders by age $11 .^{1,2}$ Despite the clinical focus on depression, youth anxiety disorders also are important because they are precursors to later development of depression and are comor-

Funding: This study was supported by the Robert Wood Johnson Foundation.

Conflict of interest: none declared.

Corresponding author: Ardis L. Olson, MD, Department of Pediatrics, HB 7450, Dartmouth Hitchcock Medical Center, Lebanon, NH 03756-0001 (E-mail: Ardis.L.Olson@dartmouth.edu). 
bid with depression. ${ }^{3}$ In one primary care setting, approximately $8.5 \%$ of adolescents interviewed had anxiety or depression, but only $22 \%$ of those had been recognized or treated by a clinician. ${ }^{4}$

Clinicians have relied largely on the interview to screen for these issues. Many adolescents report concerns and risks they wish to discuss that are not addressed during health visits. ${ }^{5}$ Multiple issues and limited time during the visit are barriers to detection of both health risk behaviors and emotional problems by interview. Screening adolescents for health behavior risks before a visit is advocated widely as a means to more consistently determining which teenagers are at high risk because of social behaviors. ${ }^{6,7}$ Recently, the US Preventive Services Task Force has recommended formal screening for depression in primary care. ${ }^{8}$ Although depression has a major impact on the adolescent population and merits screening, anxiety also causes significant morbidity. The variety of anxiety disorders means screening has limited effectiveness, ${ }^{9}$ and only recently have brief screens for generalized anxiety been available that can be utilized with adolescents in primary care.

Comprehensive screening determines who is "at risk" for depression and anxiety disorders as well as what other concerns, social risks, and assets coexist in these adolescents. The Dartmouth Healthy Teens Project has provided primary care clinicians of the Clinicians Enhancing Child Health practicebased research network with the support to conduct this type of comprehensive screening using a handheld computer during the preventive wellchild visit. ${ }^{10}$

The primary purpose of this study was to use data collected via this method to describe the pattern of mental health-related concerns, protective and social risk factors reported by adolescents during routine well-child visits in primary care settings, and their occurrence among teens who screen positive for either depression or anxiety using brief validated measures. In addition, the practicality of electronic mental health screening is discussed.

\section{Patients and Methods \\ Study Design and Population}

This cross-sectional study describes the reported concerns, health risks, behaviors, and assets of a population of adolescents attending preventive well-child visits. Data are from the Dartmouth Healthy Teens Screener, a personal digital assistant (PDA)-based questionnaire that quickly and effectively screens adolescents for health risks in primary care practices while providing clinicians with a road map for tailored counseling based on the adolescent's readiness to change key health risk behaviors.

The PDA health screener was administered as part of clinical care to adolescents aged 11 to 21 years old $(\mathrm{N}=2535)$ who attended preventive wellchild visits in 13 pediatric and family medicine primary care practices in Vermont and New Hampshire over 18 months (2008 to 2009). These practices are located in small communities with populations varying from 1000 to 50,000 people, all of which are part of the Clinicians Enhancing Child Health primary care practice-based research network. (All practices continue to use this screener 3 years after the data deadline for this study.) Data were utilized by the provider during the visit and de-identified data were transmitted regularly to an central electronic database. Full descriptions of the methodology used for the development and confirmation of the PDA-based screener as an effective tool already have been published. ${ }^{10-12}$

\section{Measures: The Healthy Teens Screener}

The Healthy Teens Screener is an electronic questionnaire that employs a branching algorithm, allowing adolescents to answer between 60 and 90 health behavior questions before a formal preventive well-child visit. The screener comprehensively covers health, mental health, and social issues relevant to adolescents. The adolescents can indicate from a list of emotional and physical issues which ones are of concern to them that they would like to discuss further with a clinician.

Since the last report of the Healthy Teens Screener, the screener has been enhanced with the addition of validated, 2-question depression and anxiety screens as well as a strength-based section (the latter after consultation with Paula Duncan of the University of Vermont ${ }^{13}$ ). The inclusion of strengthbased questions allows determination of the role of protective factors in an adolescent's risk for depression and anxiety.

Using the screener, for a patient to screen positive, that is, to be "at risk" for depression or anxiety, they had to have a score of $\geq 3$ of 6 on the brief depression and anxiety measures: the 2-question Patient Health Questionnaire (PHQ-2) and Gen- 
eralized Anxiety Disorder scale (GAD-2), respectively. Each of the questionnaires has 2 questions scored from 0 to $3(0=$ "none of the time," $1=$ "some of the time," 2 = "half of the time," and $3=$ "all the time"). The 2 questions are each summed on a scale of 0 to 6 , with totals $\geq 3$ scored as positive. These methods of screening for depression and anxiety with the above cut points have been validated previously; the PHQ-2 has a sensitivity of $79 \%$ and a specificity of $86 \%$ for any depressive disorder, and the GAD-2 has a sensitivity of $86 \%$ and a specificity of $83 \%$ for generalized anxiety disorder. ${ }^{14-18}$ The PHQ-2 has been validated in adolescent populations as well. ${ }^{19}$

The 4 strength-based questions were summed for this analysis, creating a single "Asset" category to show how protective factors were related to depression and anxiety symptoms in adolescents. Questions related to substance use (alcohol, tobacco, marijuana, and illicit drug use) were scored together as one risk factor because of the frequent overlap in these behaviors.

\section{Data Analysis}

The Healthy Teens Screener has two formats: one for younger teens (11 to 14 years old) and one for older adolescents (15 to 21 years old). For the purpose of this study, only questions represented in both iterations were used, allowing for the development of a congruent dataset among the entire study population. The dataset use for the current analysis was limited to 11 to 18 year olds $(\mathrm{N}=$ 2184) because of the small numbers in older age groups. Dartmouth Medical School's institutional review board, the Committee for the Protection of Human Subjects at Dartmouth College, approved the study protocol.

For this report, items that potentially are associated with increased likelihood of a positive screen for depression or anxiety were determined from literature review. ${ }^{20-24}$ The next step was to determine the items' association with screening positive for depression or anxiety at the bivariate level. In addition to the strength-based questions, 2 questions about familial and peer psychosocial support were asked. The assets and psychosocial support questions together constitute the protective factors, shielding the adolescents from depression and anxiety.

Our data analysis explored the role that the protective and social risk factors played in deter- mining who screens positive for depression and anxiety. First, bivariate $t$ tests and $\chi^{2}$ tests were used to establish the set of questions that were associated with positive screens of depression and anxiety. Unadjusted and adjusted logistic regression analyses were then conducted using all the symptoms identified by the bivariate methods, establishing which protective or social risk factors were individual, significant predictors for either screening positive for depression or screening positive for anxiety. All statistical tests were performed using SPSS 18.0 (IBM, Chicago, IL).

\section{Results}

Overall, at the preventive well-child visits analyzed in this study, $4.8 \%$ of adolescents in the study population had positive PHQ-2 screens and 6.3\% had positive GAD-2 screens. The demographics of the population who completed the Healthy Teens Screener are provided in Table 1.

\section{Variation in Adolescents' Concerns}

The proportion of patients having each concern was not high for any one risk factor except for substance use; $23 \%$ of the study population reported having used a substance during the month before the well-child visit, with rates varying from $13 \%$ of those in eighth grade to $61 \%$ of those in 12 th grade. The worries that the teenagers wished to discuss with the doctor changed with advancing age. Figure 1 shows that as teenagers became older 7 concerns were expressed less often whereas 3 concerns (trouble sleeping, feeling tired, and being stressed) increased.

In addition to variation by age, we found differences by sex. Specifically, high school-aged girls were more likely than their male counterparts to have several concerns. During high school (14 to 17 years old), girls were significantly $(P<.05)$ more likely to have questions or concerns related to trouble sleeping, feeling tired, and being stressed. They were also more likely to report a protective factor: parents who listen to them (Table 2).

\section{Protective and Social Risk Factors}

In the unadjusted model, all the protective and social risk factors except age were associated with screening positive for anxiety or depression. Girls had an increased likelihood of having increased 


\begin{tabular}{|c|c|}
\hline Variable & Patients, n (\%) \\
\hline \multicolumn{2}{|l|}{ Age } \\
\hline 11 & $128(5.9)$ \\
\hline 12 & $258(11.8)$ \\
\hline 13 & $273(12.5)$ \\
\hline 14 & $400(18.3)$ \\
\hline 15 & $351(16.1)$ \\
\hline 16 & $369(16.9)$ \\
\hline 17 & $299(13.7)$ \\
\hline 18 & $106(4.9)$ \\
\hline \multicolumn{2}{|l|}{ Sex } \\
\hline Female & $1162(53.2)$ \\
\hline Male & $1022(46.8)$ \\
\hline \multicolumn{2}{|l|}{ Grade } \\
\hline 5 th & $48(2.2)$ \\
\hline 6th & $185(8.5)$ \\
\hline 7 th & $230(10.5)$ \\
\hline 8th & $316(14.5)$ \\
\hline 9th & $399(18.3)$ \\
\hline 10th & $330(15.1)$ \\
\hline 11th & $356(16.3)$ \\
\hline 12 th & $298(13.6)$ \\
\hline After high school & $3(0.1)$ \\
\hline Not in school & $19(0.9)$ \\
\hline \multicolumn{2}{|l|}{ Social risk factors } \\
\hline \multicolumn{2}{|l|}{$\begin{array}{l}\text { Do you have any questions or are you worried about the following items and wish to talk } \\
\text { to the doctor about them? }\end{array}$} \\
\hline Trouble sleeping & $159(7.3)$ \\
\hline Feeling tired a lot & $243(11.1)$ \\
\hline Feeling stressed often & $234(10.7)$ \\
\hline Your anger or temper & $125(5.7)$ \\
\hline About fitting in or belonging & $41(1.9)$ \\
\hline Headaches & $80(3.7)$ \\
\hline Does anyone in your family drink or take drugs so much it worries you? & $99(4.5)$ \\
\hline Ever used illicit substances (alcohol, tobacco, or other drugs) & $506(23.2)$ \\
\hline \multicolumn{2}{|l|}{ Protective factors } \\
\hline Do you volunteer in school, church, or community?* & $527(24.1)$ \\
\hline Do you feel like you belong to a group (family, friends, or teammates)?* & $1596(73.1)$ \\
\hline Do you feel more independent as you get older?* & $1994(91.3)$ \\
\hline Do you feel like you are good at doing anything in particular (math, theater, or sports)?* & $2018(92.4)$ \\
\hline Do you have at least one friend to talk with about problems? & $2059(94.3)$ \\
\hline Do you think your parent(s) or guardian(s) usually listen to you and take you seriously? & $1964(89.9)$ \\
\hline \multicolumn{2}{|l|}{ Assets Reported, n (score) } \\
\hline 0 & $23(1.1)$ \\
\hline 1 & $101(4.6)$ \\
\hline 2 & $517(23.7)$ \\
\hline 3 & $1045(47.8)$ \\
\hline 4 & $452(20.7)$ \\
\hline \multicolumn{2}{|l|}{ Positive screen for depression ( $\geq 3$ on PHQ-2) or anxiety ( $\geq 3$ on GAD-2) } \\
\hline Depression & $104(4.8)$ \\
\hline Anxiety & $138(6.3)$ \\
\hline
\end{tabular}

*These 4 questions were scored as together as assets.

PHQ-2, 2-question Patient Health Questionnaire; GAD-2, 2-question Generalized Anxiety Disorder scale. 
Figure 1. Percentage of self-reported protective or social risk factors by age.

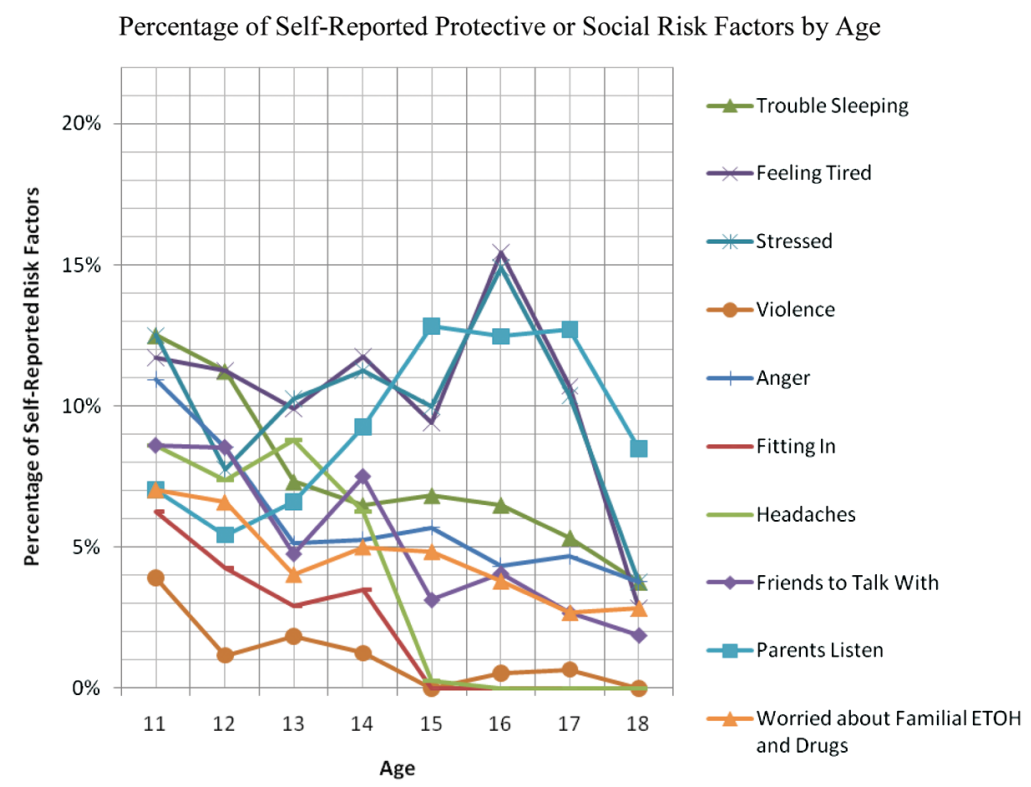

depression (5.6\% of girls were positive for depression versus $3.2 \%$ for boys) and anxiety symptoms ( $8.5 \%$ girls versus $3.5 \%$ of boys).

Table 2. Incidence of Protective and Social Risk Factors for Depression and Anxiety by Age with Significant Differences Between Female and Male Adolescents*

\begin{tabular}{|c|c|c|c|}
\hline Factor by Age & Girls (\%) & Boys (\%) & Significance \\
\hline \multicolumn{4}{|c|}{ Trouble sleeping } \\
\hline 14 years & 9.0 & 4.0 & .044 \\
\hline 15 years & 9.9 & 3.9 & .034 \\
\hline 16 years & 8.7 & 3.7 & .057 \\
\hline 17 years & - & - & - \\
\hline \multicolumn{4}{|l|}{ Feeling tired } \\
\hline 14 years & - & - & - \\
\hline 15 years & 14.1 & 5.0 & .005 \\
\hline 16 years & 20.9 & 8.6 & .001 \\
\hline 17 years & 14.1 & 6.6 & .040 \\
\hline \multicolumn{4}{|l|}{ Stressed } \\
\hline 14 years & 15.1 & 7.5 & .018 \\
\hline 15 years & 16.3 & 3.9 & .000 \\
\hline 16 years & 20.9 & 7.4 & .000 \\
\hline 17 years & 15.3 & 4.4 & .002 \\
\hline \multicolumn{4}{|l|}{ Parents listen ${ }^{\dagger}$} \\
\hline 14 years & - & - & - \\
\hline 15 years & 19.2 & 6.7 & .001 \\
\hline 16 years & 17.0 & 6.7 & .004 \\
\hline 17 years & - & - & - \\
\hline
\end{tabular}

*Student's $t$ tests were used establish these differences.

${ }^{\dagger}$ Indicates variable is a protective factor.

一, insignificant differences.
Because many of these factors coexist, the adjusted model (Table 3) informs us of significant independent associations with depressive or anxiety-related symptoms. In the adjusted model, social risk factors that were significant $(P<.05)$ predictors for a positive screen of depression included substance use (adjusted odds ratio [AOR], 2.05); stress (AOR, 3.59); anger (AOR, 1.94); and worries about family alcohol and drug use (AOR, 2.69). Among protective factors, that is, those that reduce the risk of depression, age (AOR, 0.87 for younger patients); having parents who listen (AOR, 0.34); and having more assets (AOR, 0.65) were significant.

Significant $(P<.05)$ predictors of screening positive for anxiety were included the substance use (AOR; 1.97), stress (AOR, 6.10); anger (AOR, 2.31); trouble sleeping (AOR, 1.75); and the sex of the adolescent (AOR, 1.87 for girls). Although having parents who listen was still a significant protective factor for anxiety (AOR, 0.49), the remaining assets were not significant.

\section{Discussion}

Results from this cross-sectional study indicate that adolescents who screen positive for depression or anxiety are more likely to be concerned about and wish to discuss a variety of symptoms related to their mental health. 
Table 3. Multivariate Regression Models for Depression and Anxiety

\begin{tabular}{|c|c|c|c|c|}
\hline Variable & Unadjusted OR (95\% CI) & Significance & Adjusted OR (95\% CI) & Significance \\
\hline \multicolumn{5}{|l|}{ Depression } \\
\hline Trouble sleeping & $5.52(3.45-8.82)$ & .000 & $1.75(0.95-3.21)$ & .071 \\
\hline Feeling tired & $4.32(2.80-6.68)$ & .000 & $1.04(0.58-1.89)$ & .897 \\
\hline Stressed & $8.70(5.75-13.17)$ & .000 & $3.59(2.12-6.07)$ & .000 \\
\hline Anger & $7.96(4.95-12.82)$ & .000 & $1.94(1.07-3.52)$ & .029 \\
\hline Fitting in & $5.92(2.75-12.75)$ & .000 & $1.52(0.56-4.13)$ & .414 \\
\hline Headaches & $2.65(1.29-5.47)$ & .008 & $0.98(0.39-2.50)$ & .971 \\
\hline Familial ETOH* & $6.57(3.86-11.18)$ & .000 & $2.69(1.45-5.00)$ & .002 \\
\hline Substance use & $3.14(2.10-4.67)$ & .000 & $2.05(1.24-3.40)$ & .005 \\
\hline Assets & $0.57(0.46-0.71)$ & .000 & $0.65(0.51-0.83)$ & .001 \\
\hline Talk with friends ${ }^{\dagger}$ & $0.23(0.13-0.40)$ & .000 & $0.55(0.27-1.11)$ & .093 \\
\hline Parents listen & $0.13(0.08-0.19)$ & .000 & $0.34(0.21-0.56)$ & .000 \\
\hline Age & $1.00(0.90-1.11)$ & 1.000 & $0.87(0.77-0.98)$ & .023 \\
\hline Sex & $1.94(1.27-2.96)$ & .002 & $1.37(0.87-2.17)$ & .180 \\
\hline \multicolumn{5}{|l|}{ Anxiety } \\
\hline Trouble sleeping & $5.80(3.81-8.83)$ & .000 & $1.75(1.03-2.98)$ & .038 \\
\hline Feeling tired & $5.04(3.45-7.38)$ & .000 & $1.08(0.66-1.78)$ & .759 \\
\hline Stressed & $10.05(6.94-14.55)$ & .000 & $6.10(3.94-9.43)$ & .000 \\
\hline Anger & $9.81(6.41-15.04)$ & .000 & $2.31(1.36-3.93)$ & .002 \\
\hline Fitting in & $4.93(2.36-10.27)$ & .000 & $0.85(0.32-2.26)$ & .748 \\
\hline Headaches & $2.73(1.44-5.18)$ & .002 & $1.53(0.68-3.42)$ & .301 \\
\hline Familial ETOH* & $3.69(2.14-6.37)$ & .000 & $1.44(0.77-2.68)$ & .251 \\
\hline Substance use & $3.04(2.14-4.32)$ & .000 & $1.97(1.27-3.05)$ & .003 \\
\hline Assets & $0.68(0.56-0.83)$ & .000 & $0.82(0.65-1.02)$ & .069 \\
\hline Talk with friends ${ }^{\dagger}$ & $0.32(0.18-0.56)$ & .000 & $0.60(0.31-1.18)$ & .138 \\
\hline Parents listen & $0.17(0.12-0.26)$ & .000 & $0.49(0.31-0.78)$ & .003 \\
\hline Age & $1.04(0.95-1.13)$ & .462 & $0.96(0.87-1.07)$ & .471 \\
\hline Sex & $2.49(1.69-3.66)$ & .000 & $1.87(1.24-2.83)$ & .003 \\
\hline
\end{tabular}

*Worried about familial alcohol or drug use.

${ }^{\dagger}$ Have friends to talk with about problems.

CI, confidence interval; OR, odds ratio.

Note: Items in bold type highlight which adjusted ORs have $\mathrm{p}<.05$.

Using the Healthy Teens Screener, adolescents "at risk" for depression or anxiety are not only identified in an efficient manner, but associated health concerns and strengths are also pinpointed. Rather than using questionnaires that only assess depression or anxiety status, tools such as the Healthy Teens Screener that also assess protective and social risk factors can help clinician evaluation. Clinicians will have data on both somatic and emotional concerns, and this data may be predictive of later mental illness (or early signs of later mental illness).

Strengths of this study are the ease of use and functionality of the screener for practitioners and that the screening occurred during routine wellchild visits rather than screening only when mental health issues are suspected. In addition, the screener collected information about a wide range of protective and social risk factors for depressive and anxiety-related symptoms, providing the opportunity to understand more fully the context of and address the somatic and emotional symptoms.

Last, the inclusion and assessment of protective factors of the mental health status of adolescents contributed an additional dimension to this investigation. By assessing protective factors, we have shown that the presence of strengths and assets reduce the likelihood of depression and anxiety-related symptoms being present. Not only do the protective factors reduce the likelihood of screening positive for depressive or anxiety-related symptoms, but by identifying and quantifying them, clinicians may be able to work 
with their patients and the patients' families to enhance protective factors.

In accordance with previous studies, ${ }^{25-28}$ this study found positive associations between depressive symptoms and many somatic and emotional concerns. Reporting of primary care mental health screening is limited and it typically highlights only older populations. ${ }^{29,30}$ The logic behind assessing protective factors' role in depression and anxiety diagnoses is sound, but ours is the first study explicitly looking at protective factors' contribution to whether adolescents screen positive for depression and anxiety. Although there is some overlap between the somatic and emotional risk profiles of depression and anxiety, our results show that the protective and social risk factors indicate that it may be important to screen for both disorders, considering anxiety's considerable impact.

Although there are mutual factors that predict a positive screen of depression and anxiety (substance use, stress, and anger), our analyses show that adolescents' worries about family drug and alcohol use are important to assess because they may be predictive of depression. The anxiety analysis shows a different pattern of risk factors, with female sex and sleep issues being the unique concerns that warrant particular attention by clinicians.

Expanding on the US Preventive Services Task Force's recommendation to screen all adolescents 11 to 18 years old for only depression, the results from this study suggest that a screener including anxiety helps primary care physicians more fully understand mental health issues. Routine formal screening is important because there is a risk that the somatic complaints may be the provider's primary focus during brief well-child visits.

The results indicate the importance of understanding the potential role of shared risk factors in the development of both depression and anxiety as well as the importance of comprehensive screening. Without screening for depression and anxiety, primary care physicians will be more apt to elicit and address only the somatic and emotional complaints (or not hear them at all). Screening before a visit will allow primary care physicians to collect more information during the interview portion of the well-child visit, to spend more time on concerns specific to each child, and to refer to specialists appropriately and promptly, as needed.
This study was limited by several factors. The study included a nonrandom sample of almost all white patients, and the results need to be duplicated using other cultures, demographics, and geographic regions, but the strength is that this is data from clinical community care settings. It will be important to replicate this work in other settings. We used a tool that detects a population with symptoms of depression and anxiety. With these symptoms, adolescents are more likely to have a clinical diagnosis. In fact, even without a clinical diagnosis of depression or anxiety, those who screen positive on the PHQ-2 are more likely to have other emotional and somatic symptoms. Our study is limited in that the PHQ-2 and GAD-2 screen for significant levels of symptoms, but are not diagnostic of a disorder. However, high levels of symptoms, even without a clinical disorder, were found in our study to be associated with a variety of other emotional and somatic symptoms. This is consistent with other works using the 9-item PHQ with adolescents, ${ }^{4}$ among whom symptoms in the absence of a Diagnostic and Statistical Manual of Mental Disorders, Fourth Edition, diagnosis were associated with significant emotional distress. Last, the sample size was too small to examine the $2 \%$ of adolescents who screen positive for both depression and anxiety.

\section{Conclusions}

Comprehensive primary care mental health screening that considers both anxiety and depression while including strength-based and psychosocial support questions is a helpful adjunct to clinical practices and has been done routinely by using an electronic format at the point of care. Because certain somatic and emotional concerns can precede depression and anxiety, routine electronic screening for these issues during wellchild visits is suggested.

Screening from early adolescence onward will increase the likelihood of primary care physicians catching the early signs, symptoms, and documented risks of depression and anxiety. Using an electronic screener bolsters primary care physician-patient interactions by giving the provider data before the visit and freeing up time spent interviewing to discuss specific concerns. The electronic medium bolsters communication, while tailoring to the technology-based generation, demon- 
strating that these aims need not be mutually exclusive in clinical care.

Zsolt Nagykaldi, PhD, helped to develop the PDA screening tool. We thank the physicians and staff of the following practices in our network whose efforts made PDA screening a reality: Ammonoosuc Community Health Services, Whitefield, NH; Dartmouth Hitchcock Pediatric Practices (Bedford, NH; Concord, NH; Lebanon, NH; Plymouth, NH); Exeter Pediatric Associates, Exeter, NH; Green Mountain Pediatrics, Bennington, VT; Montpelier Health Center, Montpelier, VT; Robert A. Mesropian Center for Community Care at Alice Peck Day Memorial Hospital, Lebanon, NH; Springfield Pediatrics, Springfield, VT; Upper Valley Pediatrics, Bradford, VT; and Wolfeboro Pediatrics, Wolfeboro, NH.

\section{References}

1. Kessler RC, Berglund P, Demler O, Jin R, Merikangas KR, Walters EE. Lifetime prevalence and ageof-onset distributions of DSM-IV disorders in the National Comorbidity Survey Replication. Arch Gen Psychiatry 2005;62(6):593-602.

2. Merikangas KR, He JP, Burstein M, et al. Lifetime prevalence of mental disorders in U.S. adolescents: results from the National Comorbidity Survey Replication-Adolescent supplement (NCS-A). J Am Acad Child Adolesc Psychiatry 2010;49(10):980-9.

3. Chavira DA, Stein MB, Bailey K, Stein MT. Comorbidity of generalized social anxiety disorder and depression in a pediatric primary care sample. J Affect Disord 2004;80:163-71.

4. Richardson LP, Russo JE, Lozano P, McCauley E, Katon W. Factors associated with detection and receipt of treatment for youth with depression and anxiety disorders. Acad Pediatr 2010;10(1):36-40.

5. Klein JD, Wilson KM. Delivering quality care: adolescents' discussion of health risks with their providers. J Adolesc Health 2002;30(3):190-5.

6. Hagan JF, Shaw JS, Duncan PM, eds. Bright Futures: Guidelines for Health Supervision of Infants, Children, and Adolescents. Third Edition. Elk Grove Village, IL: American Academy of Pediatrics; 2008.

7. Katon W, Russo J, Richardson L, McCauley E, Lozano P. Anxiety and depression screening for youth in a primary care population. Ambul Pediatr 2008; $8(3): 182-8$.

8. Williams SB, O'Connor EA, Eder M, Whitlock EP. Screening for child and adolescent depression in primary care settings: a systematic evidence review for the U.S. Preventive Services Task Force. Pediatrics 2009;123(4):e716-35.

9. Gardner W, Lucas A, Kolko DJ, Campo JV. Comparison of the PSC-17 and alternative mental health screens in an at-risk primary care sample. J Am Acad Child Adolesc Psychiatry 2007;46(5):611-8.

10. Olson AL, Gaffney CA, Hedberg VA, Gladstone GR. Use of inexpensive technology to enhance ad- olescent health screening and counseling. Arch Pediatr Adolesc Med 2009;163(2):172-7.

11. Olson AL, Gaffney CA, Hedberg VA, et al. The healthy teen project: tools to enhance adolescent health counseling. Ann Fam Med 2005;3(Suppl 2):S63-5.

12. Olson AL, Gaffney CA, Lee PW, Starr P. Changing adolescent health behaviors: the healthy teens counseling approach. Am J Prev Med 2008;35(5 Suppl): S359-64.

13. Duncan PM, Garcia AC, Frankowski BL, et al. Inspiring health adolescent choices: a rationale for and guide to strength promotion in primary care. J Adolesc Health 2007;41(6):525-35.

14. Kroenke K, Spitzer RL, Williams JB, Löwe B. The Patient Health Questionnaire somatic, anxiety, and depressive symptom scales: a systematic review. Gen Hosp Psychiatry 2010;32(4):345-59.

15. Kroenke K, Spitzer RL, Williams JB. The Patient Health Questionnaire-2: validity of a two-item depression screener. Med Care 2003;41(11):1284-92.

16. Löwe B, Kroenke K, Gräfe K. Detecting and monitoring depression with a two-item questionnaire (PHQ-2). J Psychosom Res 2005;58(2):163-71.

17. Spitzer RL, Kroenke K, Williams JB, Löwe B. A brief measure for assessing generalized anxiety disorder: the GAD-7. Arch Intern Med 2006;166(10):1092-7.

18. Kroenke K, Spitzer RL, Williams JB, Monahan PO, Löwe B. Anxiety disorders in primary care: prevalence, impairment, comorbidity, and detection. Ann Intern Med 2007;146(5):317-25.

19. Richardson LP, Rockhill C, Russo JE, et al. Evaluation of PHQ-2 as a brief screen for detecting major depression among adolescents. Pediatrics 2010;125(5): e1097-103.

20. American Psychiatric Association. Diagnostic and statistical manual of mental disorders. Revised Fourth Edition. Washington, DC: Author; 2000.

21. Kroenke K, Spitzer RL, Williams JBW, et al. Physical symptoms in primary care: predictors of psychiatric disorders and functional impairment. Arch Fam Med 1994;3:774-9.

22. Kroenke K, Jackson JL, Chamberlin J. Depressive and anxiety disorders in patients presenting with physical complaints: clinical predictors and outcome. Am J Med 1997;103(5):339-47.

23. Haug TT, Mykletun A, Dahl AA. The association between anxiety, depression, and somatic symptoms in a large population: the HUNT-II study. Psychosom Med 2004;66(6):845-51.

24. Katon W, Sullivan M, Walker E. Medical symptoms without identified pathology: relationship to psychiatric disorders, childhood and adult trauma, and personality traits. Ann Intern Med 2001; 134(9):917-25.

25. Kopec MT, Randel J, Naz B, et al. Using the Guidelines for Adolescent Preventive Services to estimate adolescent depressive symptoms in school-based health centers. Fam Med 2010;42(3):193-201. 
26. Hallfors DD, Waller MW, Ford CA, Halpern CT, Brodish PH, Iritani B. Adolescent depression and suicide risk: association with sex and drug behavior. Am J Prev Med 2004;27(3):224-31.

27. Kubik MY, Lytle LA, Birnhaum AS, Muray DM, Perry CL. Prevalence and correlates of depressive symptoms in young adolescents. Am J Health Behav 2003;27(5):546-53.

28. Rushton JL, Forcier M, Schectman RM. Epidemiology of depressive symptoms in the National Longi- tudinal Study of Adolescent Health. J Am Acad Child Adolesc Psychiatry 2002;41(2):199-205.

29. Eisenberg D, Gollust SE, Golberstein E, Hefner JL. Prevalence and correlates of depression, anxiety, and suicidality among university students. Am J Orthopsychiatry 2007;77(4):534-42.

30. Kinnunen P, Laukkanen E, Kylmä J. Associations between psychosomatic symptoms in adolescence and mental health symptoms in early adulthood. Int J Nurs Pract 2010;16(1):43-50. 